\title{
Continuous observation of seismic wave velocity and apparent velocity using a precise seismic array and ACROSS seismic source
}

\author{
Atsushi Saiga ${ }^{1 *}$, Koshun Yamaoka $^{2}$, Takahiro Kunitomo ${ }^{3}$, and Toshiki Watanabe ${ }^{4}$ \\ ${ }^{1}$ Graduate School of Science, Nagoya University, Nagoya 468-8602, Japan \\ ${ }^{2}$ Earthquake Prediction Research Center, Earthquake Research Institute, University of Tokyo, Tokyo 113-0032, Japan \\ ${ }^{3}$ Tono Geoscience Center, Japan Nuclear Cycle Development Institute, Gifu 509-5132, Japan \\ ${ }^{4}$ Research Center for Seismology, Volcanology and Disaster Mitigation, Nagoya University, Nagoya 468-8602, Japan
}

(Received September 28, 2005; Revised May 28, 2006; Accepted May 29, 2006; Online published September 16, 2006)

\begin{abstract}
We report the results of continuous monitoring — using a seismometer array-of the travel time of seismic waves generated by an ACROSS artificial seismic source. The seismometer array, which was deployed in a surface vault located $2.4 \mathrm{~km}$ from the source, recorded both direct $P$ - and $S$-waves and refracted $P$ - and $S$ waves that traveled along a velocity boundary between the granite basement and overlying sedimentary rocks. We analyzed temporal variation in differential travel time and apparent velocity for these phases for a period of 1 month and found significant temporal variation in the differential travel time. Most of the variation can be attributed to changes in environmental conditions, such as atmospheric temperature and rainfall. Variation is even observed in the seismogram that is located $50 \mathrm{~m}$ from the vibration source, although much smaller variation is observed in the vibration of the foundation to which the source is attached. The spectral study revealed that the effects of temperature and rainfall depend strongly on the frequency range used by ACROSS and that a large variation occurs in the $15-$ to $20-\mathrm{Hz}$ range, especially between 17 and $20 \mathrm{~Hz}$. The environmental effect on the temporal variation is comparable to the record of refracted $S$ waves and that of a distance of $50 \mathrm{~m}$, whereas a larger variation was observed in the direct $S$ wave. This result shows that the signal is affected by the environmental change near the vibration source. The environmental effect can be drastically reduced when the signal from the $15-$ to $20-\mathrm{Hz}$ range is eliminated in the analysis.
\end{abstract}

Key words: Active monitoring, seismometer array, ACROSS, temporal varia tion, spectral analysis.

\section{Introduction}

Various methods have been tested for their sensitivity and reliability to detect changes in the propagation properties of seismic waves related to changes in the travel medium (see Crampin et al., 1990; Li et al., 1998; Niu et al., 2003). Changes in stress and fluid distribution, for example, can affect propagation properties, such as the velocity, attenuation, dispersion and anisotropy of seismic waves.

Laboratory experiments indicate that the velocity of elastic waves in rocks is strongly dependent upon the nature of the stress field (Birch, 1960). This relationship is attributed to the increasing stiffness and seismic velocity of rocks under high stress that results from the closure of structural defects such as cracks and joints. Semenov (1969) monitored the velocity of direct $P$ - and $S$-waves and recorded reductions in the $V p / V s$ ratio immediately prior to large earthquakes; these observations are the forerunners to the dilatancy-diffusion theory (Nur, 1972; Sholtz et al., 1973). In contrast to these findings, McEvilly and Johnson (1974) failed to detect any variation in the $V p / V s$ ratio

\footnotetext{
*Now at Institute of Seismology and Volcanology, Faculty of Sciences, Kyushu University, Shimabara, Nagasaki 855-0843, Japan.

Copyright (c) The Society of Geomagnetism and Earth, Planetary and Space Sciences (SGEPSS); The Seismological Society of Japan; The Volcanological Society of Japan; The Geodetic Society of Japan; The Japanese Society for Planetary Sciences; TERRAPUB
}

prior to moderate magnitude earthquakes in central California even though seismic velocity was carefully monitored using quarry blasts of precisely controlled timing. These authors concluded that in order to successfully monitor velocity changes, additional careful studies would be required within incipient source regions using wave paths that propagate through the source region. The question of whether it is practically possible to measure velocity changes resulting from changes in tectonic stress, however, remains controversial.

The scattering of seismic waves is a potentially effective method of detecting changes in physical properties, such as attenuation properties, as it can be used to sample an entire crustal volume, such as a rupture zone. Sato (1988) reported a significant increase in the scattering and attenuation of seismic waves prior to and following the 1984 Western Nagano earthquake $(\mathrm{Ms}=6.8)$. Similarly, Hiramatsu et al. (2000) recorded an increase in coda $\mathrm{Q}^{-1}$ following the 1995 Hyogoken-Nanbu earthquake and attributed this change to variations in static stress that resulted from the earthquake. Changes in the anisotropy of seismic waves have also been detected in association with seismic events. For example, temporal changes in the time delay of split shear waves have been observed immediately prior to earthquake events (e.g. Crampin et al., 1990; Bokelmann and Harjes, 2000). This phenomenon has been explained by applying a theory of extensive dilatancy anisotropy (Crampin, 1987) in 
which the differential stress field dictates the preferential alignment of newly formed or growing cracks. Saiga et al. (2003) reported temporal variation in the time delay of split shear waves resulting from static stress changes associated with a moderate magnitude earthquake. These observations demonstrate the sensitivity of the propagation properties of seismic waves to changes in applied stress; however, these researchers were not always able to analyze events of similar source parameters. Successive earthquakes with identical source parameters, such as hypocenter and focal mechanism, are ideally required for accurate monitoring of the propagation properties of seismic waves.

An accurate and continuous experiment is necessary to detect temporal variations in seismic velocity within the earth's crust. Reasenberg and Aki (1974) conducted an experiment that involved measuring the travel time of an airgun shot signal over a distance of $200 \mathrm{~m}$. They detected a variation in travel time of $1 \mathrm{~ms}$, which can be correlated to the effects of the earth's tide. Yamamura et al. (2003) conducted long-term observations using a piezoelectric transducer over a distance of $12 \mathrm{~m}$, and also detected variations in travel time that show a strong correlation with the earth's tide. Furumoto et al. (2001) demonstrated that characteristic temporal changes in seismic wave velocity can be related to tidal stress and a secular stress accumulation resulting from repeated observation explosions. There are, however, few studies that have undertaken the accurate and continuous long-term monitoring of seismic velocity using an artificial source.

A system that uses an artificial source and which is capable of continuous and precise monitoring of the propagation properties of seismic waves within the crust has already been developed (Kumazawa and Takei, 1994; Yamaoka et al., 2001). This system is called the Accurately Controlled Routinely Operated Signal System (ACROSS). The vibrator in the ACROSS system generates a signal with a centrifugal force of a rotating eccentric mass. The rotation is precisely synchronized to global positioning system (GPS) timing, which provides large numbers of stacks for data that are also sampled in synchrony with GPS timing at a receiver. Given a 1-week stacking, the signal generated by the ACROSS vibrator can be detected at seismic stations more than $100 \mathrm{~km}$ from the source (Yamaoka and Ikuta, 2004; Yoshida et al., 2004). The vibrator used in this study can produce a maximum force of $2 \times 10^{5} \mathrm{~N}$, but one can barely feel the vibration even at a distance of $10 \mathrm{~m}$.

Ikuta et al. (2002) monitored temporal variations in travel time for 15 months using a surface-deployed ACROSS source and seismometers at depths of $800 \mathrm{~m}$ and $1700 \mathrm{~m}$ in boreholes along the Nojima fault zone, which was the fault zone that ruptured during the 1995 Hyogo-ken Nanbu (Kobe) earthquake. The authors were able to measure temporal variation in the travel time of direct waves over a long period with high accuracy. Ikuta and Yamaoka (2004) detected sudden delays and subsequent gradual recovery in the travel time of direct $P$ - and $S$-waves generated by the 2000 Western-Tottori earthquake $(\mathrm{Mw}=6.6)$ and the 2001 Geiyo earthquake $(\mathrm{Mw}=6.4)$. The delays were also accompanied by an increase in shear wave splitting. These authors interpreted the measured variation as resulting from an increase in pore pressure within preferentially aligned cracks in the uppermost crust due to strong seismic shaking. The importance of this study is that the authors were able to demonstrate that seismic velocity could be monitored over a long period of time at a high accuracy.

Based on the above experiments, all of which revealed the potential of the ACROSS system, we sought to utilize seismometer arrays to detect and monitor various phases included in the ACROSS signal. Niu et al. (2003) detected a temporal variation in the seismogram of repeated microearthquakes measured by seismic stations. Their results revealed a change in the location of scatterers within a fault zone related to an aseismic deformation event. In the current study, we set out to document precise observations using a seismic array with the aim of investigating temporal variations in the seismic wave velocity and apparent velocity of various phases propagating through the crust. The observation site that we selected for this study is a research site located deep underground, where subsurface structure is well understood and where many observational facilities, such as strainmeters, groundwater wells and seismometers, are already deployed.

\section{Site Description and System Deployment \\ 2.1 Observation array}

We deployed a seismic array within a vault used for the observation of crustal deformation. As our aim was to detect temporal variations in the propagation properties of seismic waves, it was important to minimize artificial variations resulting from the observation system. We therefore deployed both seismometers and the data-logging system in the vaults, where temperature remains constant to within $0.1^{\circ} \mathrm{C}$ for the entire year. The array comprised 15

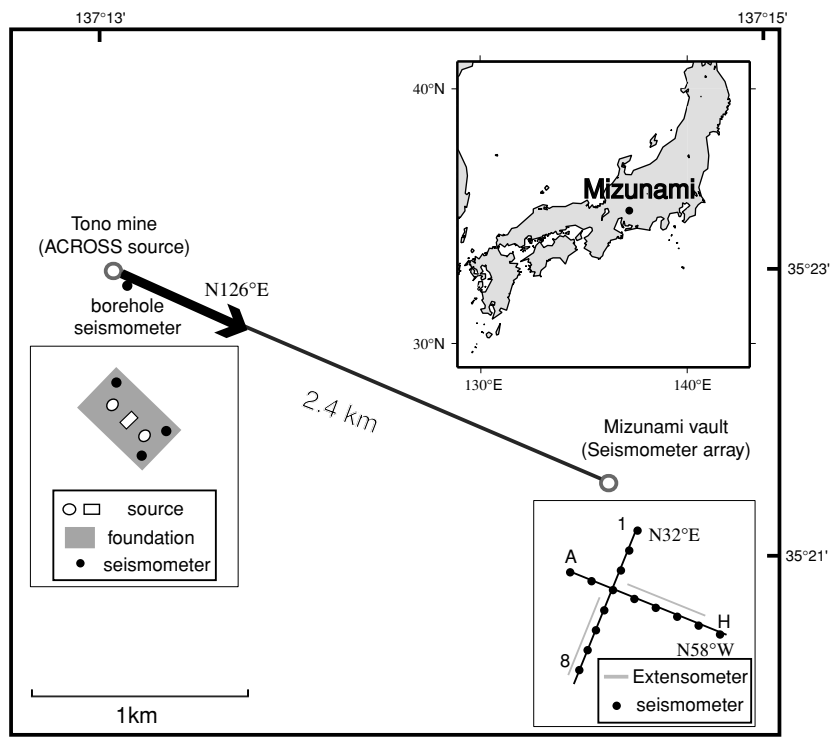

Fig. 1. Map of the experiment site. The ACROSS sources and seismometer array are deployed in the Tono mine and in a vault used for the observation of crustal deformation in Mizunami, respectively. Solid circles denote seismometers. The seismometer array is $64 \times 64 \mathrm{~m}$ at the interval of $8 \mathrm{~m}$, as a cross shape along extensometers. Open circles and an open rectangular denote three ACROSS sources, which have rotation axes in the vertical and the horizontal direction, respectively. The ACROSS sources are covered with a foundation. 
three-component velocity sensors (Mark Products Inc.; L28LBH) with a natural frequency of $4.5 \mathrm{~Hz}$ and sensitivity of $0.61 \mathrm{~V} /(\mathrm{cm} / \mathrm{s})$. The seismometers were deployed linearly at 8-m intervals in two tunnels that intersect at right angles.

The vault that housed the seismometer array is located $2.4 \mathrm{~km}$ from the ACROSS source used in this study (Fig. 1) and is constructed within Neogene sedimentary rock of the Mizunami group that overlies the Cretaceous granite basement. The ACROSS source was deployed on the surface of the Mizunami group; phases that propagate through the sedimentary rock and granite basement can therefore be monitored by the array with good resolution.

\subsection{Data logging system for array observation}

The logging system consisted of an amplifier and PC with inbuilt digitizers. The signals from the seismometers were amplified to ensure that ground noise was in excess of the quantization noise of the digitizer. The signal was digitized by A/D converters with synchronization to GPS timing. We adopted a sampling rate of $1 \mathrm{kHz}$ to reduce the effect of antialiasing filtering, although the signal of the ACROSS source was $<30 \mathrm{~Hz}$. The filter was used to reduce the noise above Nyquist frequency, which otherwise overlaps with the frequency band of the ACROSS signal through the aliasing effect. The cut-off frequency of the anti-alias filter was set at $250 \mathrm{~Hz}$, which was high enough to suppress the phase effect on the frequency band of the ACORSS signal. Delay times due to channel switching in the digitizing process and signal transmission from seismometers were measured and corrected as appropriate.

\subsection{GPS synchronization for array observation}

The signals from seismometers were digitized in synchronization with GPS timing. For synchronization, we used a 1 pulse-per-minute (PPM) code and 1 kilo-pulse-persecond (KPPS) code that were generated by a GPS clock. The data sampling, which is synchronized to the 1 KPPS code, starts every hour with synchronization to the 1 PPM code. The accuracy of the timing is kept to within $0.1 \mu \mathrm{s}$ of universal time.

As the logging system was installed approximately 200 $\mathrm{m}$ from the entrance of the vault, we had to transmit the codes to the data logger from the GPS clock that is located by the entrance. We used differential line-drivers and differential line-receivers for transmitting both the 1 PPM and 1 KPPS signals to avoid the effect of large common-mode noise. The delay time involved in transmitting signals was corrected as appropriate.

\subsection{Source deployment}

The ACROSS vibrators used in this study were deployed in the Tono mine, Gifu prefecture, Japan. Three ACROSS vibrators with different eccentric moments were deployed. The low-frequency (LF) vibrator produced a force of $2 \times 10^{5}$ $\mathrm{N}$ at a frequency of $25 \mathrm{~Hz}$, the mid-frequency (MF) vibrator produced $1.9 \times 10^{5} \mathrm{~N}$ at a frequency of $35 \mathrm{~Hz}$ and the highfrequency $(\mathrm{HF})$ vibrator produced $1.6 \times 10^{5} \mathrm{~N}$ at a frequency of $50 \mathrm{~Hz}$. The vibrator force is generated by the centrifugal force of a rotating eccentric mass and is therefore proportional to the square of the rotational velocity. The ACROSS vibrators were firmly fixed to a foundation of steel-framed concrete structure, which was constructed in a rectangular hole of dimensions 6 (length) $\times 3.5$ (width) $\times 2.3 \mathrm{~m}$ (depth).
The eccentric masses of the LF and MF units rotate around a vertical axis, whereas that of the HF unit rotates around a horizontal axis. Three accelerometers were installed on the foundation to monitor any movement.

The vibration of the source was synchronized to GPS timing (Yamaoka et al., 2001). The rotation of the mass is modulated in frequency to produce plural spectral peaks simultaneously. The modulation is repeated at a constant interval, resulting in the production of a stable force for each spectral peak.

\section{Obtaining the Transfer Functions \\ 3.1 Virtual linear vibrator}

The ACROSS vibrators generate acceleration through the rotation of eccentric mass around an axis, although linear acceleration is often useful for sources of seismic signals. In most rotational vibrators (e.g. Alekseev et al., 2005), linear acceleration is produced by rotating two motors in opposite directions. In contrast to traditional vibrators, we synthesize a linear acceleration with the data of a single rotor. The motor of the ACROSS vibrator switches its rotational direction at 1-h intervals, and we were able to synthesize a linear motion by combining the signals of two successive hours. The great advantage of this method is that we can synthesize a linear acceleration in any direction at every hour. The synthesized data $Y^{\theta}$ in the direction $\theta$ is obtained via a linear combination (Kunitomo and Kumazawa, 2004) as:

$$
Y^{\theta}(\omega)=Y^{n}(\omega) \exp (i \theta)+Y^{r}(\omega) \exp (-i \theta)
$$

where $Y^{n}$ and $Y^{r}$ are the observed spectrums when the ACROSS source rotates in clockwise and counterclockwise directions, respectively, and $\omega$ is the angular frequency of the signal.

The force at the source is also synthesized in a similar way:

$$
X^{\theta}(\omega)=X^{n}(\omega) \exp (i \theta)+X^{r}(\omega) \exp (-i \theta)
$$

where $X^{n}$ and $X^{r}$ are the spectrums of the generated force when the source rotates in clockwise and counterclockwise directions, respectively. We can then use these data to obtain transfer functions for different components of the vibrations.

\subsection{Transfer function}

The transfer function between the force generated at the source and the signal observed at the array is obtained via a deconvolution of observed waveform by source signal in the frequency domain:

$$
G_{k}^{\theta}(\omega)=Y_{k}^{\theta}(\omega) / X^{\theta}(\omega)
$$

where $G_{k}^{\theta}$ represents the transfer function of the $k$-th component of the receivers for a linear acceleration at the source in the direction $\theta$.

A waveform in the time domain is obtained by applying an Inverse Fourier Transform (IFT) to the transfer function in Eq. (3). In the operation of the ACROSS source, we used a frequency modulation technique to produce plural frequency components simultaneously. Figure 2 shows an example of these waveforms and the spectrum for a virtual 

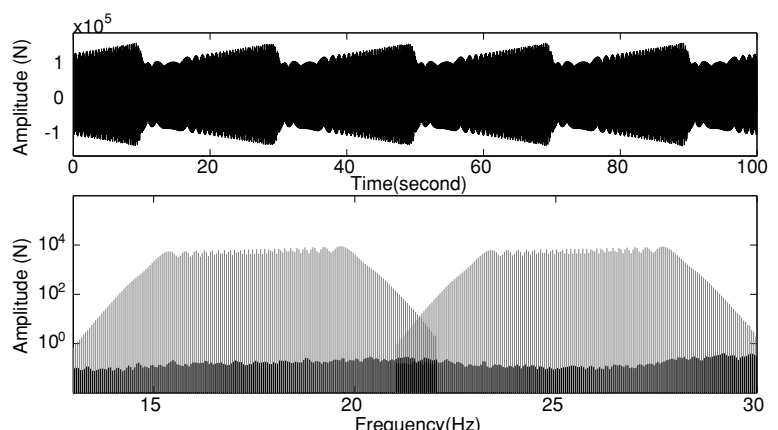

(b)
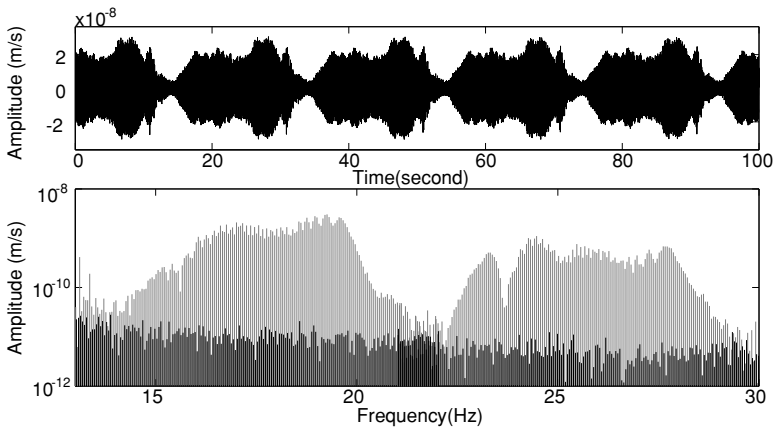

(c)
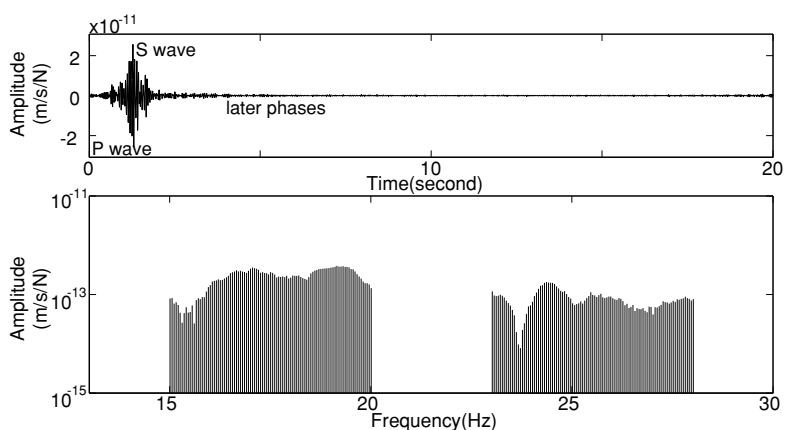

Fig. 2. (a) Theoretical force generated at the ACROSS sources: A waveform (top); the amplitude spectrum (bottom). The gray and the black lines indicate signals of the ACROSS sources and noises, respectively. (b) (Top) A record at seismometer C. (Bottom) The amplitude spectrum. (c) (Top) A transfer function between the source and seismometer $\mathrm{C}$ in the time domain. (Bottom) The transfer function in the frequency domain.

linear vibration in the radial direction; Fig. 2(a) shows a force generated at the ACROSS source. The force is calculated theoretically from the rotation velocity of the eccentric mass. Figure 2(b) shows a record at a seismometer at the center of the array for 150-h stacking. As a same modulation is repeated every $20 \mathrm{~s}$, signals from the source appear every $1 / 20 \mathrm{~Hz}$ in the frequency domain; these are called the signal components, whereas others are termed noise com-

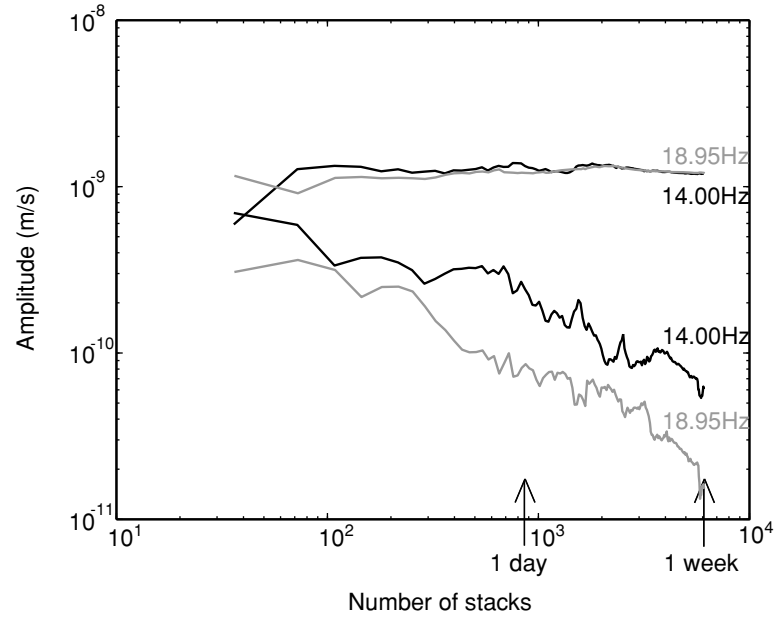

Fig. 3. Variation in the amplitude of the signal and noise components with a greater number of stacks in the first experiment. Gray lines show the signal and noise components around $18.95 \mathrm{~Hz}$; black lines show those around $14.00 \mathrm{~Hz}$. The level of signal components is almost constant in contrast to the level of noise that decreases with stacking.

ponents. The signal-to-noise ratio (SNR) can be estimated from the ratio of the amplitudes of the signal and noise components, which is about 102 in this experiment. By using the signal components for source and receivers, we can obtain transfer functions. Figure 2(c) shows the transfer function in both the frequency and time domains. The signal components with small amplitudes are omitted in the transfer function in the frequency domain. The transfer function in the time domain is calculated using these data. Several phases with large amplitudes are found in the transfer function in the time domain [Fig. 2(c)].

A signal leakage is apparent in the transfer function in the time domain, especially around $20 \mathrm{~s}$ in Fig. 2(c). The leakage may have resulted from an inappropriate selection of window function when applying the IFT. This effect also occurs in other parts of the transfer function in the time domain, and small later phases might be concealed by the leakage effect. We use cosine-taper windows in the following analysis as an optimum window function.

\section{Phase Identification and Estimation of Tempo- ral Change}

We conducted three experiments with the aim of detecting temporal variations in the travel times of waves propagating along various paths between the source and the receivers. Table 1 shows the operation parameters of the ACROSS source used in the experiments.

Table 1. Transmit parameter of the ACROSS sources.

\begin{tabular}{ccccccc}
\hline $\begin{array}{c}\text { Number of } \\
\text { experiments }\end{array}$ & $\begin{array}{c}\text { Time Period of } \\
\text { Experiments }\end{array}$ & $\begin{array}{c}\text { Source Type } \\
\text { (Rotation axis) }\end{array}$ & $\begin{array}{c}\text { Source Type } \\
\text { (Type of frequency) }\end{array}$ & \multicolumn{2}{c}{ Time Period of Center Frequency } & Moduration \\
FM [second $]$ & Generated Force [N] \\
\hline 1 & 1 week & Vertical & MF & 10 & 21.45 & $0.6 \times 105 \sim 0.9 \times 105$ \\
& Vertical & LF & 10 & 16.50 & 2.5 & $0.6 \times 105 \sim 1.2 \times 105$ \\
2 & 4 days & Horizontal & HF & 20 & 30.00 & 10.00 \\
& Vertical & LF & 20 & 15.01 & 5.00 & $0.3 \times 105 \sim 1.3 \times 105$ \\
3 & 1 month & Vertical & MF & 20 & 25.53 & 2.50 \\
& Vertical & LF & 20 & 17.52 & 2.50 & $0.7 \times 105 \sim 1.3 \times 105$ \\
\hline
\end{tabular}


$(\mathrm{V}-\mathrm{V})$

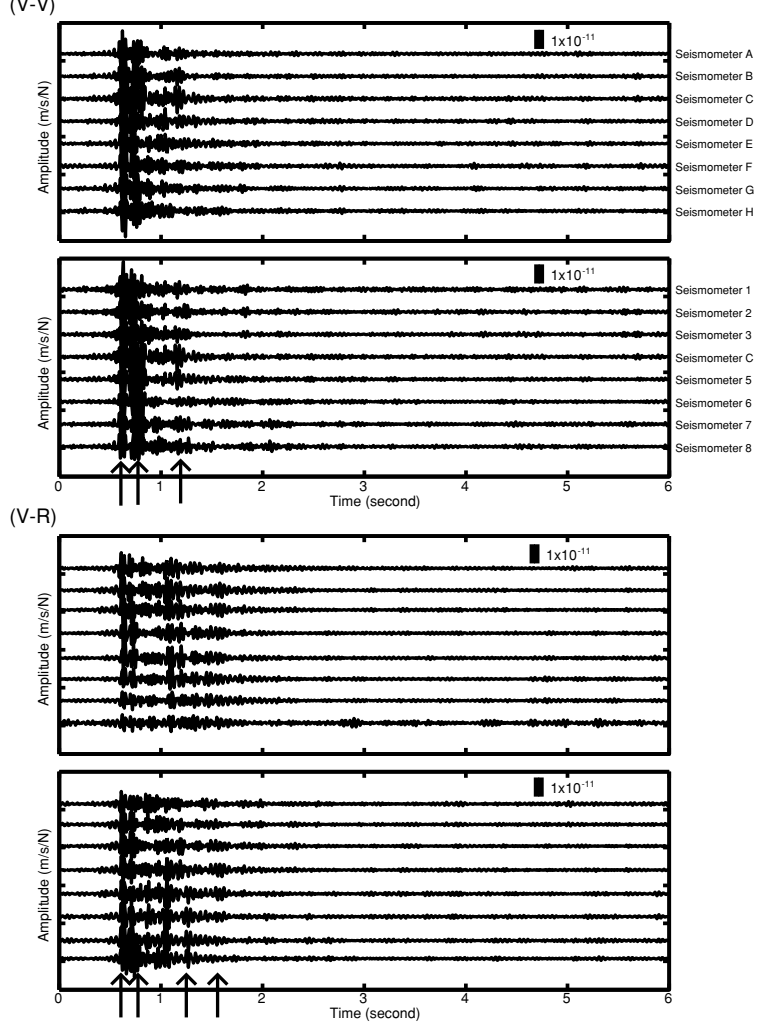

(T-T)

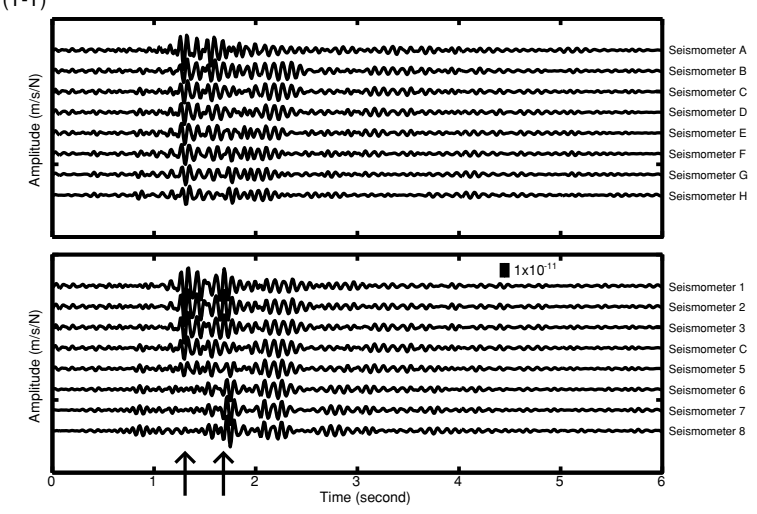

(R-V)

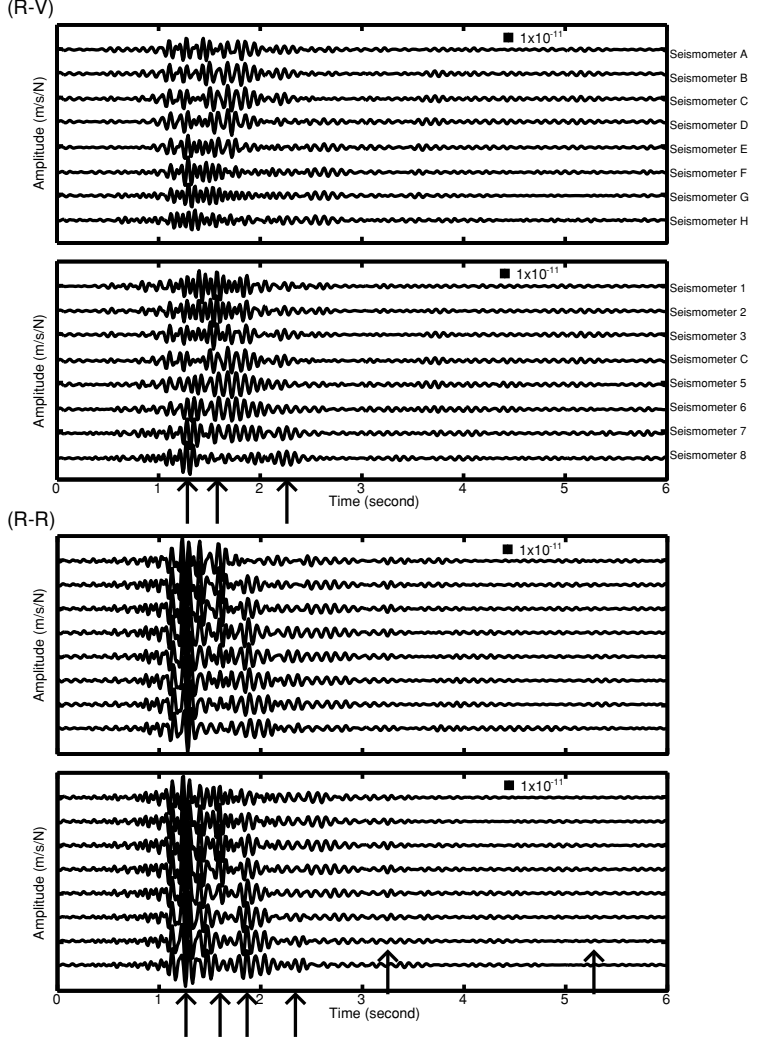

Fig. 4. Three component waveforms at the array for each component of the vibration. (V-V) Waveform at the vertical component for the vertical component of vibration. (V-R) Waveform at the radial component for the vertical component of vibration. (R-V) Waveform at the vertical component for the radial component of vibration. (R-R) Waveform at the radial component for the radial component of vibration. (T-T) Waveform at the transverse component for the transverse component of vibration. The frequency band 10.01-20.01 $\mathrm{Hz}$ is used for the radial and the transverse component of vibrations. A broader frequency band, which ranges from 20.00 to $40.00 \mathrm{~Hz}$, is used for the vertical component of vibrations. Arrows denote an arrival of marked phases.

In the first experiment, we examined the efficiency of stacking to obtain a SNR that is large enough to enable the identification of phases. Figure 3 shows the variation in the amplitude of signal and noise components versus the number of stacks obtained in the first experiment. We were able to obtain a SNR of approximately 100 for each signal component by stacking the data for a period of 1 week.

In the second experiment, a broad-frequency band was used for the source signal. The use of a broadband signal as a source produced superior time resolution of phases in the transfer functions. In this experiment we operated the source with changing rotation direction to obtain the transfer function corresponding to linear vibrations of various directions. For radial and vertical vibrations, the trans- fer functions had larger amplitudes in the vertical and radial components than in the transverse component. For the transverse vibrations, the transfer function had a large amplitude in the transverse component. Working under the assumption that $P$ - and $S$-wave behavior in an isotropic homogeneous medium can be applied in this site, we separated the $P$ - and $S V$-waves from the $S H$-wave in the transfer functions. Wherever possible, we attempted to detect various phases as in an isotropic homogeneous medium.

Figure 4 shows the three-component waveforms at the array for each component of the vibrations. In the vertical component of vibrations, four phases are evident at approximately $0.6,0.8,1.2$ and $1.6 \mathrm{~s}$ of travel time. Four more phases are also evident in the radial component of vibra- 

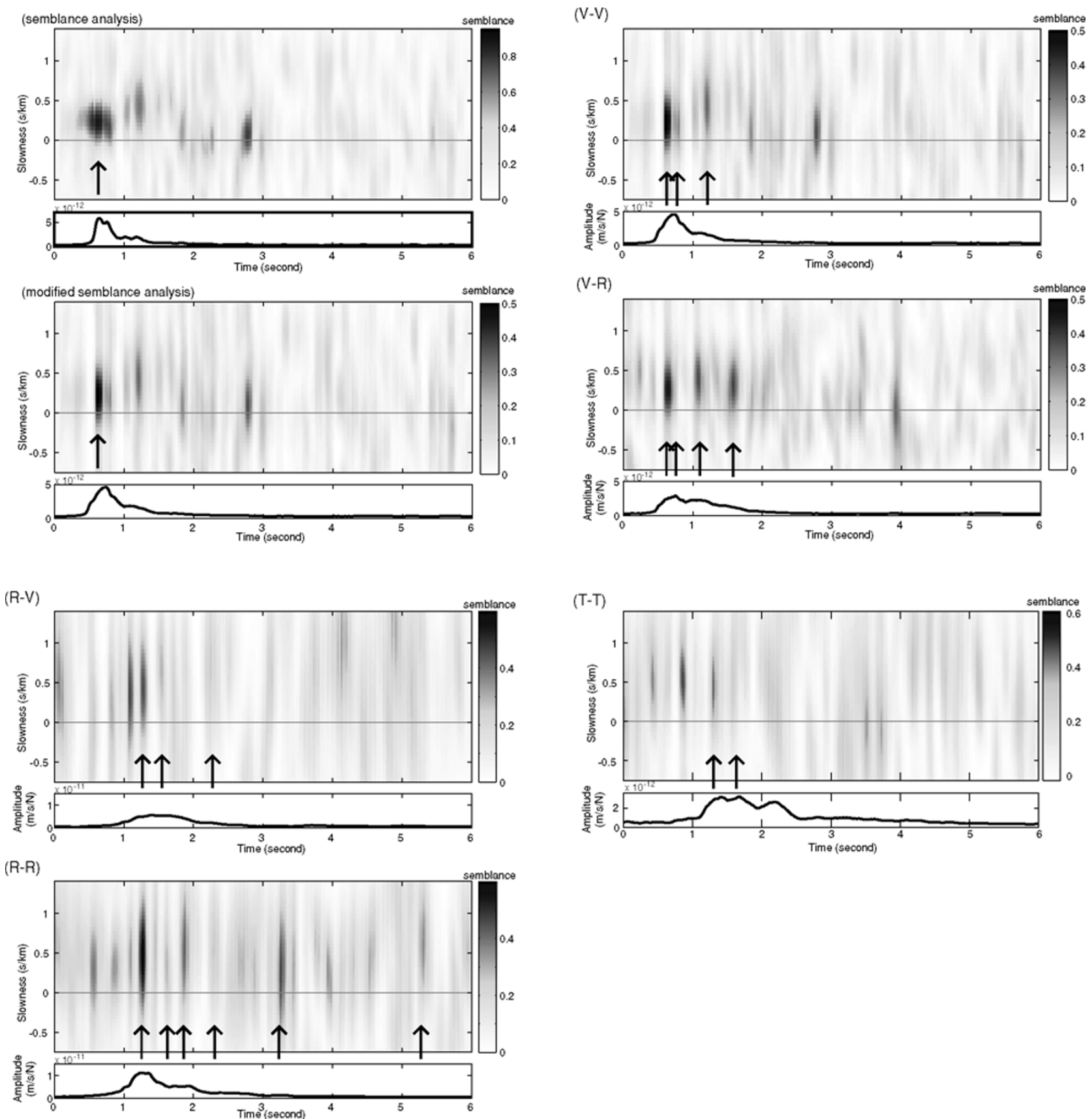

Fig. 5. (a) An example of the semblance map calculated in this study. (Top) A semblance map for an ordinary semblance analysis representing the radial component waveform at the array for the radial component of vibration. Time windows in the calculation are $0.1 \mathrm{~s}$ in both the numerator and denominator. (Bottom) A semblance map for a modified semblance analysis. Time windows in the calculation are $0.1 \mathrm{~s}$ in the numerator and 0.3 $\mathrm{s}$ in the denominator. (b) Semblance map for the modified semblance analysis at each component waveform at the array for the vertical, radial and transverse component of vibration. Symbols for figures denote the component waveform at the array and the component of the vibration, as shown in Fig. 5.

tions at approximately 1.9, 2.3, 3.2 and $5.2 \mathrm{~s}$. In the transverse component of vibration, there were two clear phases at approximately 1.3 and $1.6 \mathrm{~s}$. In the following sections we investigate these ten phases to determine their wave properties.

\subsection{Semblance analysis of the ACROSS signal}

To extract the spatially coherent component of the ACROSS signal, we used the semblance method (Neidell and Tanar, 1971) as a coherency measure for multi-channel data. The semblance value $S_{j n}$, as a function of time and apparent slowness, is expressed as

$$
S_{j n}=\frac{\sum_{j=-J / 2}^{J / 2}\left(\sum_{k=1}^{K} g_{j k}\left(t_{j}, \alpha_{n}\right)\right)^{2}}{K \cdot \sum_{j=-J / 2}^{J / 2} \sum_{k=1}^{K} g_{j k}\left(t_{j}, \alpha_{n}\right)^{2}}
$$

where $g_{j k}$ denotes a wavefield at the $j$-th sample and $k$-th seismometer, and $J$ and $K$ are the number of samples in the select window and seismometers, respectively. In this equation, $g_{j k}\left(t_{j}, \alpha_{n}\right)$ is obtained using an inverse Fourier 

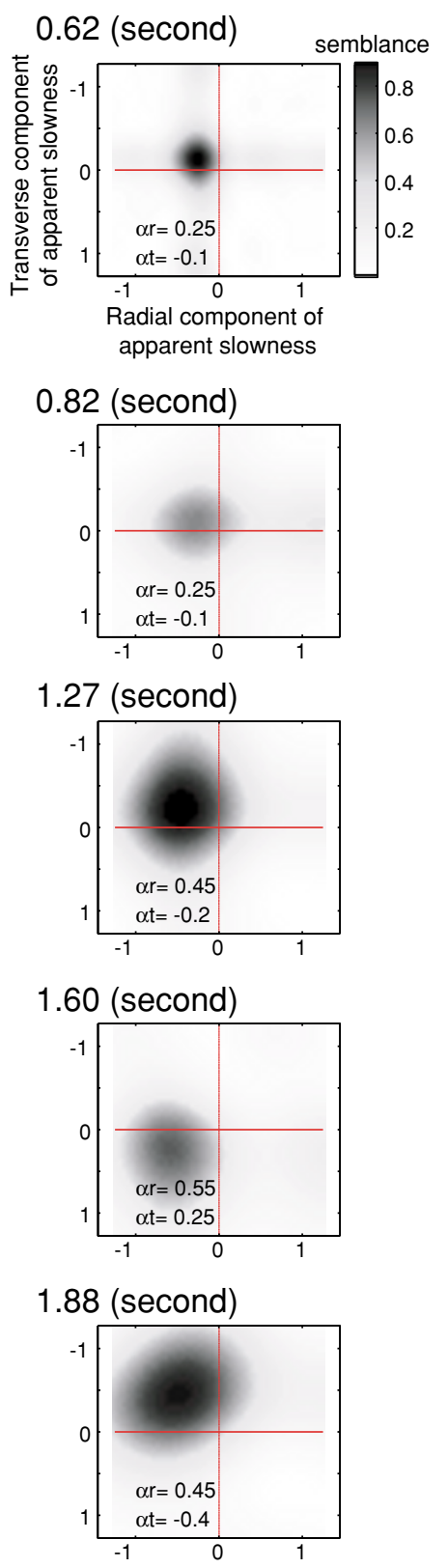
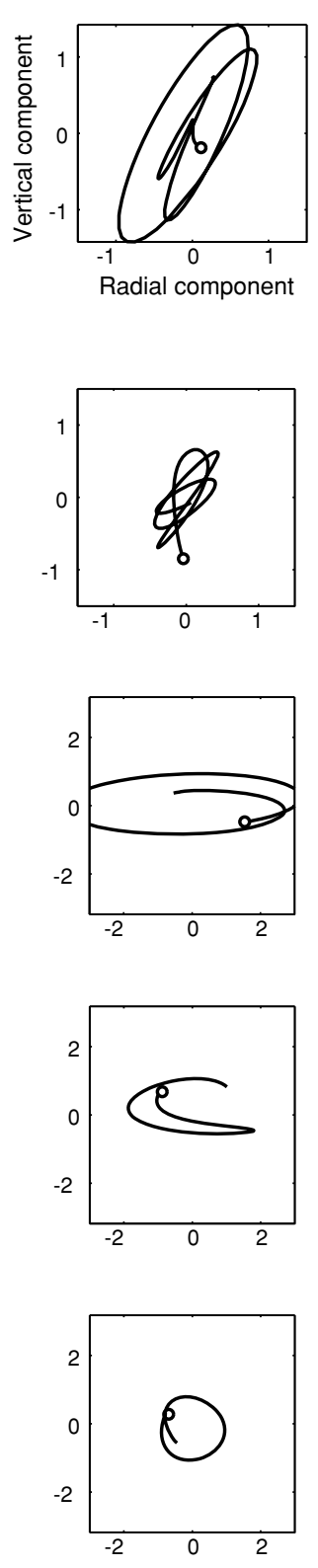
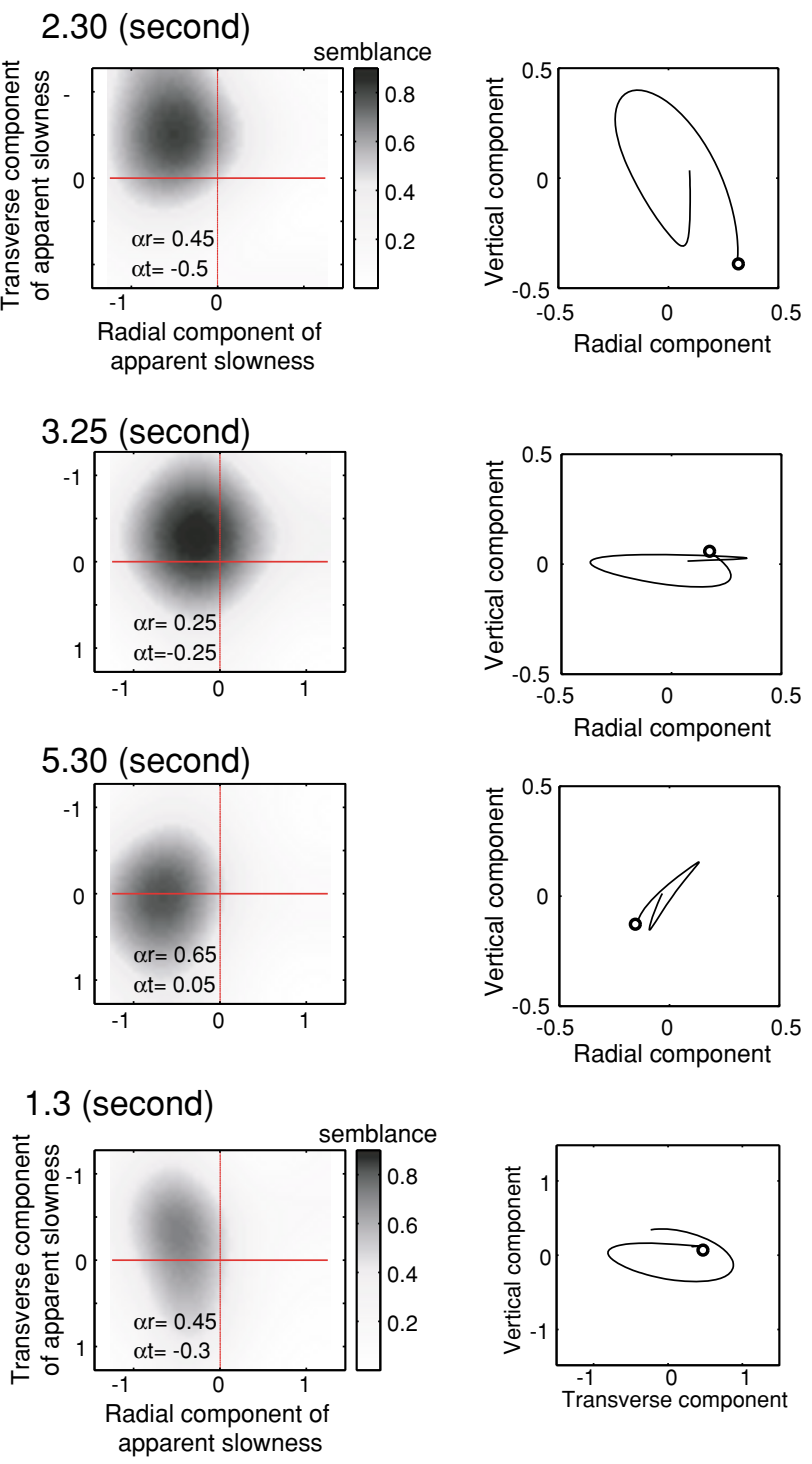

1.6 (second)

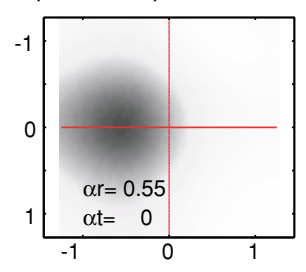

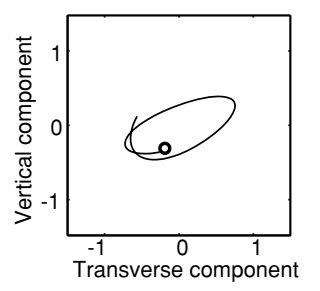

Fig. 6. (Left) Semblance map for an ordinary semblance analysis as a function of the radial and transverse component of apparent slowness for each phase. Negative of the radial component of apparent slowness shows an arrival from the source direction. (a) $0.62,0.82,1.27,1.60,1.88,2.30,3.25$ and $5.30 \mathrm{~s}$ for the vertical and radial components of the vibrated directions. (b) 1.30 and $1.60 \mathrm{~s}$ for the transverse component of the vibrated directions. (Right) Particle motion of each phase. Circle indicates a start point of particle motion.

transformation as,

$$
g_{j k}\left(t_{j}, \alpha_{n}\right)=\operatorname{IFT}\left\{G_{l k} \cdot \exp \left(i \omega_{l}\left(t_{j}-\alpha_{n} \cdot x_{k}\right)\right)\right\}
$$

where $x_{k}$ is the position vector of the seismometer, $\alpha_{n}$ is apparent slowness and $t_{j}$ is time. IFT denotes inverse the Fourier transformation.

Figure 5 provides an example of a semblance map calculated in this study. The figure shows the time-slowness plot of the semblance value for the radial component at the receivers for the case of radial vibration at the source.
The axis of slowness is oriented in the receiver-source direction. There are many peaks of high semblance values even where signal amplitude is small. In this experiment we used a signal with a limited frequency band; side-lobes are therefore inevitable in the resultant transfer function in the time domain. As such, a traditional semblance calculation, in which the information of amplitude vanishes, is inappropriate. Consequently, we introduce a modified semblance method that takes into account the signal amplitude, and we expand the time window of the denominator in the 
(a)

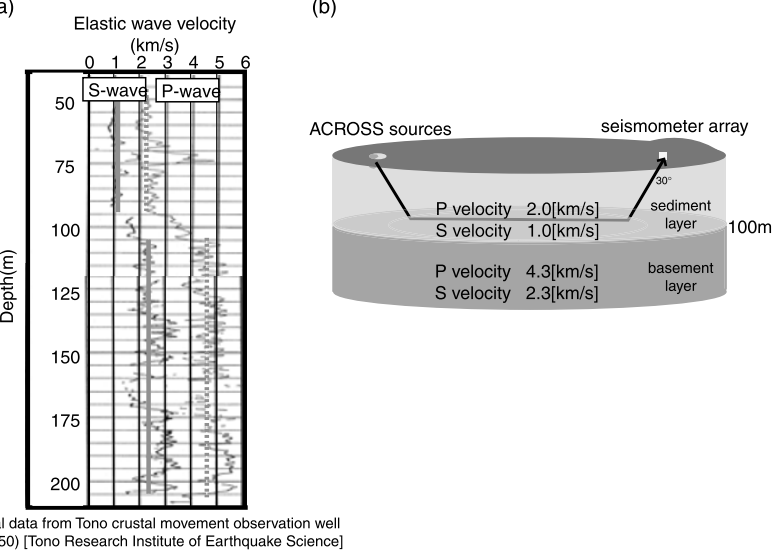

Fig. 7. (a) Velocity structure under the array site down to $200 \mathrm{~m}$. There is a pronounced change in the elastic wave boundary around $100 \mathrm{~m}$. (b) Schematic figure of a velocity structure under the experiment site.

semblance calculation. This operation enables us to detect a coherent signal with a large amplitude. We determined the time window of the numerator and the denominator for 0.1 $\mathrm{s}$ and $0.3 \mathrm{~s}$, respectively. Figure 5(a) also shows the results of the modified semblance calculations. The lower figure of the semblance map shows the averaged amplitude of denominators in a window of time and space. The averaged amplitude serves as a weighting in the modified semblance calculation. A clear peak in semblance values is evident at $0.6 \mathrm{~s}$ in the modified semblance map; the weighting suppresses spurious peaks in semblance values derived from the traditional one, such as the peak around $0.6 \mathrm{~s}$. In this operation, we can clearly determine the travel time and apparent slowness of each phase.

Figure 5(b) shows the results of the modified semblance calculations for each component of vibrations, and Fig. 6 shows a contour map of semblance values as a function of the radial and transverse components of apparent slowness $\alpha_{r}$ and $\alpha_{t}$ at the arrival time of each phase. Negative $\alpha_{r}$ indicates an arrival of waves from the source direction. For the $P$ - and $S V$-components, coherent phases are apparent at $0.62 \mathrm{~s}$ and $1.27 \mathrm{~s}$, with apparent velocities of approximately $3.7 \mathrm{~km} / \mathrm{s}$ and $2.0 \mathrm{~km} / \mathrm{s}$, respectively. It appears that these phases did not arrive directly from the direction of the source but arrived from a direction slightly to the north of the source. Coherent phases are also apparent at 0.82, 1.60, 1.88 and $2.30 \mathrm{~s}$. with apparent velocities of about 3.7, 1.7, 1.7 and $1.5 \mathrm{~km} / \mathrm{s}$, respectively. Other coherent signals with large apparent velocities occur at 3.25 and $5.30 \mathrm{~s}$. For the $\mathrm{SH}$-component, we detected coherent phases at 1.3 and 1.6 $\mathrm{s}$, with apparent velocities of 1.8 and $1.8 \mathrm{~km} / \mathrm{s}$, respectively.

\subsection{Identification of waves}

Geophysical logging exploration was used to investigate velocity structures to a depth of several hundred meters at the experiment site (TRIES, 2001; Fig. 7). A pronounced velocity boundary exists at about a depth of $100 \mathrm{~m}$, which is the boundary between the granitic basement and overlying sedimentary layers. The velocity of the sedimentary layers is approximately $2.0 \mathrm{~km} / \mathrm{s}$ for $P$-waves and $1.0 \mathrm{~km} / \mathrm{s}$ for $S$-waves, while basement granite records velocities of 4.3 $\mathrm{km} / \mathrm{s}$ for $P$-waves and $2.3 \mathrm{~km} / \mathrm{s}$ for $S$-waves. The wave ob- served at $0.62 \mathrm{~s}$ has an apparent velocity of $3.7 \mathrm{~km} / \mathrm{s}$, which corresponds to $P$-wave velocity in the basement layer. Particle motion of the wave is in a sub-vertical direction. These results show that the wave observed at $0.62 \mathrm{~s}$ is the refracted $P$-wave traveling through basement rocks. Similarly, the wave observed at $1.27 \mathrm{~s}$ has an apparent velocity of 2.0 $\mathrm{km} / \mathrm{s}$, which corresponds to the $S$-wave velocity in basement rocks. Particle motion of the wave shows a nearly radial direction. These results indicate that the wave observed at $1.27 \mathrm{~s}$ is the refracted $S$-wave traveling through the basement rocks. The refracted wave arrived at the array a little northerly of the source direction, indicating that the boundary dips southward.

Coherent phases arriving after the refracted $P$-wave and the refracted $S$-wave are found at 0.82 and $1.60 \mathrm{~s}$, with apparent velocities of approximately $3.7 \mathrm{~km} / \mathrm{s}$ and $1.7 \mathrm{~km} / \mathrm{s}$, respectively. Although the apparent velocities of these phases are similar to those of the refracted waves, they arrived before the surface waves, which arrived at 1.88 and $2.30 \mathrm{~s}$, with apparent velocities of $1.7 \mathrm{~km} / \mathrm{s}$ and $1.5 \mathrm{~km} / \mathrm{s}$, respectively. We therefore propose that the 0.82 and 1.60 s waves are a direct $P$-wave and a direct $S$-wave, respectively. The arrival times of these phases supports this interpretation. Other coherent phases with high apparent velocity are found at 3.25 and $5.30 \mathrm{~s}$, with apparent velocities of $2.8 \mathrm{~km} / \mathrm{s}$ and $1.5 \mathrm{~km} / \mathrm{s}$, respectively. We interpret these phases to represent waves reflected from the deeper parts of the velocity boundary; however, the aperture size of the array used in this study was too small to resolve the detailed apparent velocity of these phases.

In terms of the $S H$-component, $S H$-waves are recorded at 1.3 and $1.6 \mathrm{~s}$, with apparent velocities of 1.8 and $1.8 \mathrm{~km} / \mathrm{s}$, respectively. We interpret these two phases to represent the refracted and direct $S H$-waves, respectively.

\subsection{Estimation of differential travel time and differen- tial apparent velocity}

To examine small delays or advances in travel time for these phases, we calculated the cross-spectral density between a reference waveform and an observed one. The two waveforms are extracted with the same time window. The cross-spectral density, $C_{l k}$, is calculated as follows:

$$
\begin{aligned}
G_{l k} & =\mathrm{FT}\left\{\operatorname{IFT}\left(G_{j k}\right) \cdot h_{j}\right\} \\
C_{l k} & =G_{l k}^{r e f *} \cdot G_{l k}^{o b s}(l=1, \cdots, L ; \quad k=1, \cdots, K)
\end{aligned}
$$

where FT denotes the Fourier transformation, * denotes a complex conjugate and $h_{j}$ is an applied window function. Differential travel times for a sensor and a frequency $\Delta t_{l k}(l=1, \cdots, L ; \quad k=1, \cdots, K)$ are obtained by dividing the phase difference of the cross spectrum by an angular frequency:

$$
\Delta t_{l k}=\operatorname{angle}\left(C_{l k}\right) / \omega_{l}
$$

where angle denotes a phase angle of the $C_{l k}$.

We estimated the average differential travel time and differential apparent slowness from differential travel time for all the elements of the array. Assuming that a phase arrives at the array as a plane wave, the differential travel time $\Delta \tau$ at the origin of the array and the differential apparent slow- 


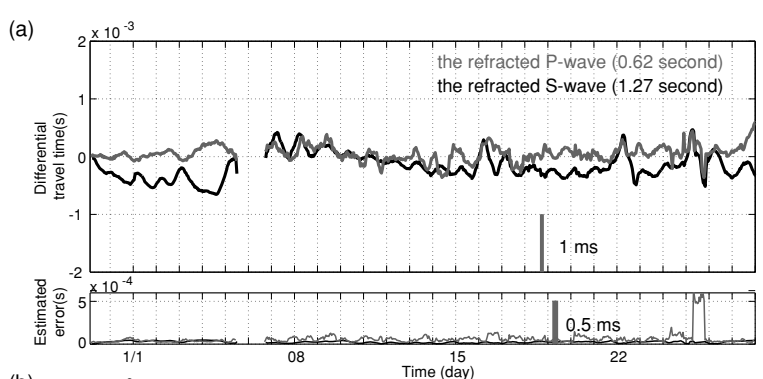

(b)
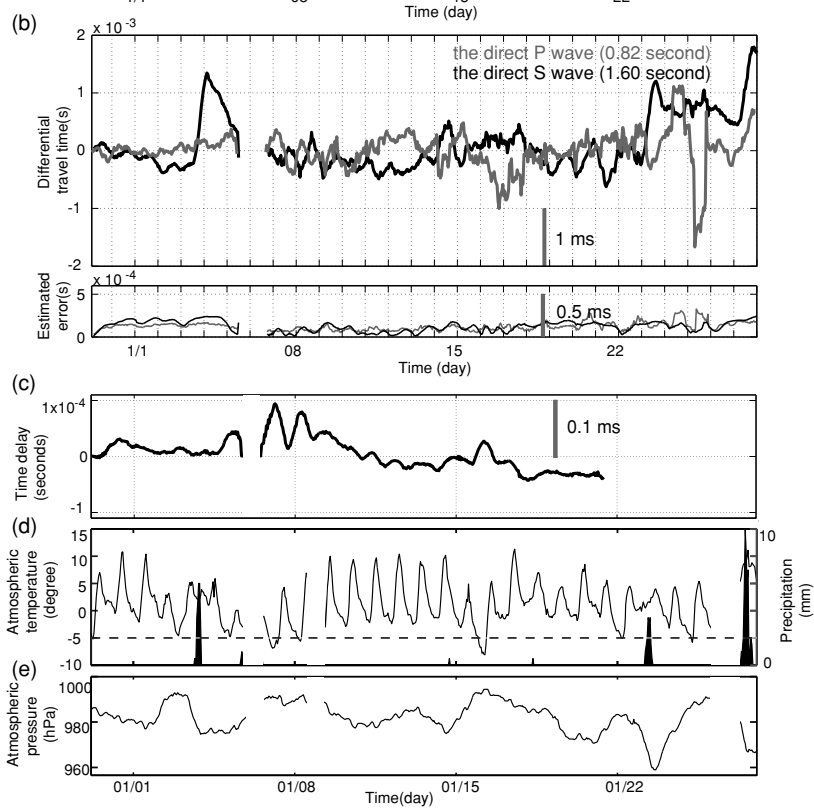

Fig. 8. Temporal variation in the differential travel time for (a) the refracted $P$-wave and the refracted $S$-wave, (b) the direct $P$-wave and the direct $S$-wave. The data stacked in $12 \mathrm{~h}$ was used for the evaluation. Error was estimated by deviations in the differential travel time from the estimated plane as an uncertainty. (c) Time delay in the radial motion of the source foundation. The time delay is expressed as the average of the delays over the frequency range that the ACROSS source generated. (d) Atmospheric temperature and precipitation, (e) Atmospheric pressure.

ness $\Delta \alpha$ are expressed as

$$
\Delta t_{l k}=\Delta \tau+\Delta \alpha \cdot x_{k}
$$

The differential apparent slowness corresponds to a change in the elastic wave velocity of the medium immediately beneath the receivers or to the incident angle of the wave that encounters the receivers. The values of $\Delta \tau$ and $\Delta \alpha$ are evaluated by the weighted least squares method with the following weighting:

$$
W_{l k}\left|C_{l k}\right|
$$

The error is estimated from the deviation of differential travel time from the estimated plane as an uncertainty.

We use data obtained by stacking for $12 \mathrm{~h}$ around each time in the observation period. Data measured at the beginning of the experiment are used as a reference. A 0.4-s hanning window was used to extract each phase.

It is difficult to estimate the variation in differential apparent slowness for phases of small amplitude. To address this problem, we spatially stacked data, focusing on a phase with appropriate phase shift, and calculated a crossspectrum density. The differential travel time is obtained by averaging the differential travel times at each frequency. (a)

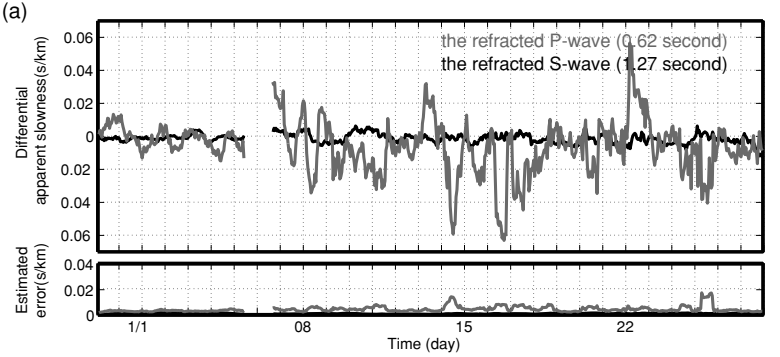

(b)

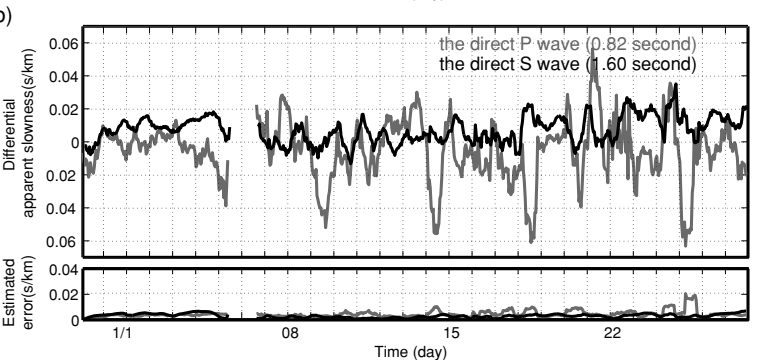

(c)

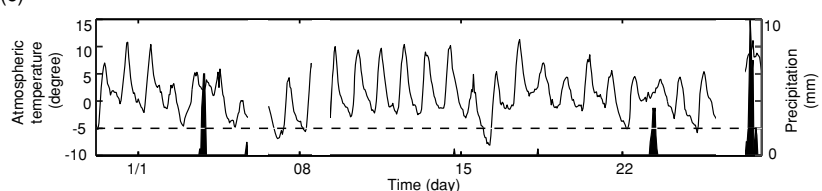

Fig. 9. Temporal variation in a differential apparent slowness for (a) the refracted $P$-wave, and the refracted $S$-wave, (b) the direct $P$-wave and the direct $S$-wave. (c) Temporal variation in the atmospheric temperature and precipitation.

\section{Temporal Variations in Travel Time and Ap- parent Velocity}

We used observational data from the seismic array to monitor differential travel time and differential apparent slowness for various phases with a high semblance value. The results for each phase are presented below.

\subsection{Refracted $P$-waves and $S$-waves}

Figure 9(a) shows temporal variation in differential apparent slowness. The variation corresponds to that of the incident angle or to the spatially heterogeneous variation of elastic wave velocity just beneath the site of array. Temporal variations in the elastic wave velocity beneath the array can result from meteorological effects as well as other factors.

We first compared the variations in travel time with variations in atmospheric temperature, precipitation and atmospheric pressure [Fig. 8(d) and 8(e)]. The variation in travel time shows a similar pattern to variations in atmospheric temperature, with delays for periods of decreasing atmospheric temperature. Large variations are observed when the temperature is less than $-5^{\circ} \mathrm{C}$. Figure 10 (a) shows a correlation between a differential travel time for the refracted $S$ wave and atmospheric temperature. In this figure we demonstrate the correlation by a solid line for the period between January 21, 900 hours, and Jan 25, 900 hours, when the atmospheric temperature is relatively low. The correlation coefficient in this period is -0.72 , although the correlation coefficient for the whole period is -0.17 . These results demonstrate that atmospheric temperature affects travel time variation. It is highly unlikely that the changes in atmospheric temperature affected the observation system 
(a)

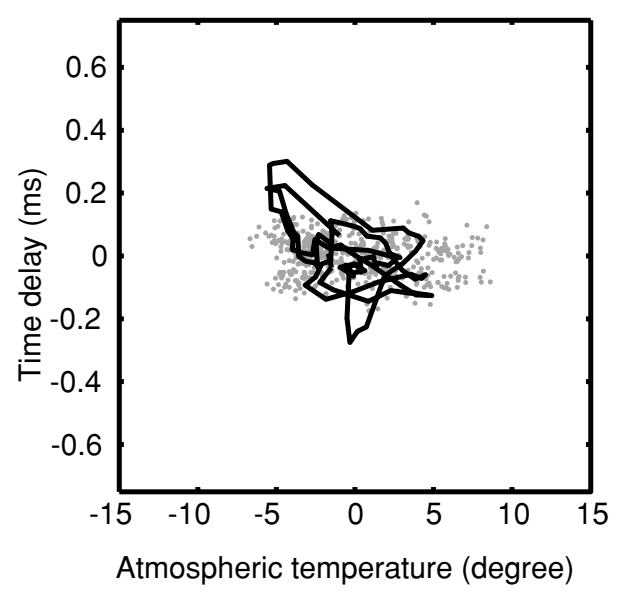

(b)

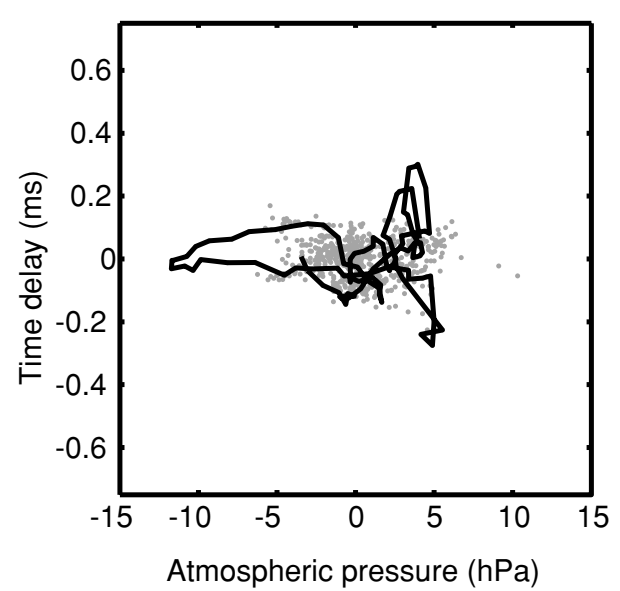

Fig. 10. (a) Correlation between a differential travel time for the refracted $S$ wave and atmospheric temperature. Each value is shown by subtracting a short period trend, which is evaluated by a moving average over 2 days. Solid line shows the correlation in a period between Jan 21 , 900 hours, and Jan 25, 900 hours, when the correlation coefficient is high. (b) Correlation between a differential travel time for the refracted $S$ wave and atmospheric pressure. Solid line shows the correlation in the period between Jan 21 9:00 and Jan 25 9:00.
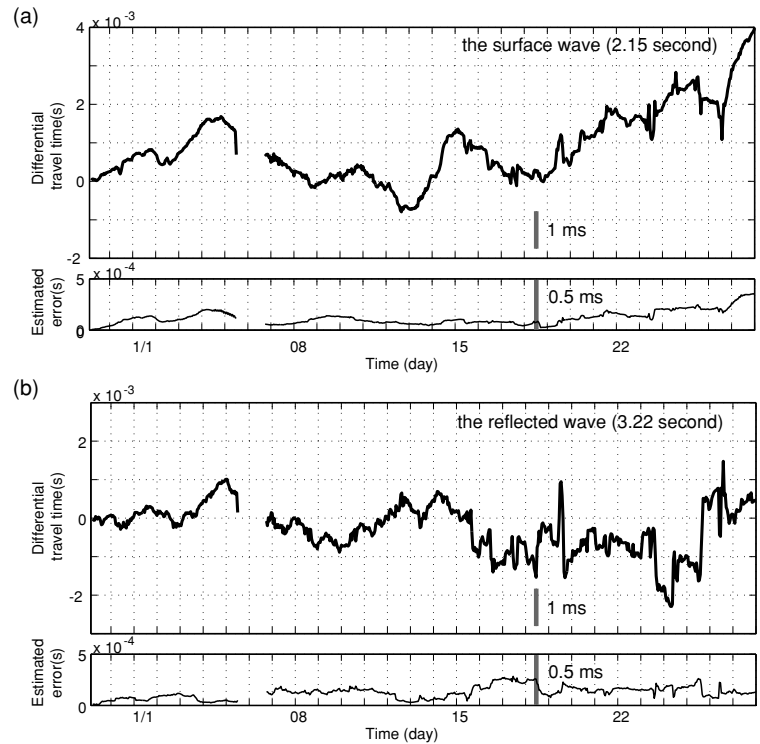

(c)

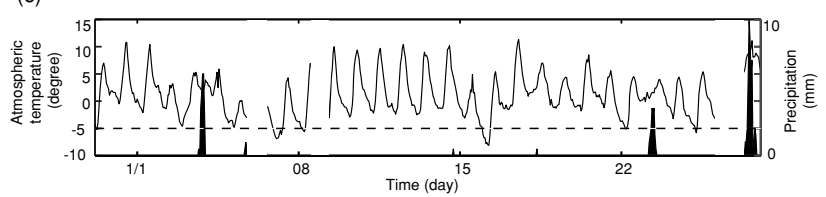

Fig. 11. (a) Temporal variation in differential travel time for the surface wave $(2.15 \mathrm{~s})$. (b) Temporal variation in differential travel time for phases with a high apparent velocity $(3.25 \mathrm{~s})$. Data were stacked for $48 \mathrm{~h}$ in these phases because the amplitude of these phases was small. (c) Temporal variation in atmospheric temperature and precipitation.

and elastic wave velocity near the array as the seismometers were deployed in a temperature-constant vault. The effects of rainfall and barometric pressure are scarcely apparent in these data [Fig. 10(b)]. The correlation coefficient of the refracted $S$ wave to barometric pressure is -0.01 .

Figure 8(a) shows the temporal variation in differential travel time for the refracted $P$ - and $S$-waves for which prop- agation properties were determined. The differential travel time shows a cyclic variation with a period of 1 day. The maximum amplitude of the variation is approximately 0.5 $\mathrm{ms}$, which corresponds to $10^{-3}$ of a velocity change. Longterm and step-like variations are also apparent, with amplitudes of approximately $0.5 \mathrm{~ms}$. The uncertainty in the differential travel time, which arises from the frequencydependent deviation, is estimated to be within $0.1 \mathrm{~ms}$, which corresponds to a precision of $10^{-4}$. The variations in velocity change observed in this experiment are therefore statistically significant.

We compared the variation in the travel time with a variation in the motion of the source foundation [Fig. 8(c)]. Although the rotation of the eccentric mass in the source is precisely controlled with reference to GPS timing, the motion (i.e. acceleration) of the source is strongly affected by changes in the elastic or inelastic properties of the ground surrounding the source. For example, if the stiffness of the surrounding ground decreases, the amplitude of the motion of the source increases. Inelastic properties such as Q may also affect the motion of the source. Saeki (2000) demonstrated that the energy emitted from the source and dissipated around the source is proportional to the phase delay of the motion of the source.

Phase variation on the source foundation shows similar patterns to variation in atmospheric temperature. This indicates that changes in atmospheric temperature affect the elastic property of the ground surrounding the source. The temporal variation in travel time observed at the array, however, is still fivefold greater than the variation that can be explained by phase variation of the source motion. No comparable pattern of temporal variation is apparent in the differential apparent slowness. The effects of daily variation in atmospheric temperature do not penetrate far into the ground, but do affect the physical properties of the ground to depths of approximately $1 \mathrm{~m}$. The observed refracted wave travels along the bedrock-sediment boundary at a depth of approximately $100 \mathrm{~m}$ for most of the distance 

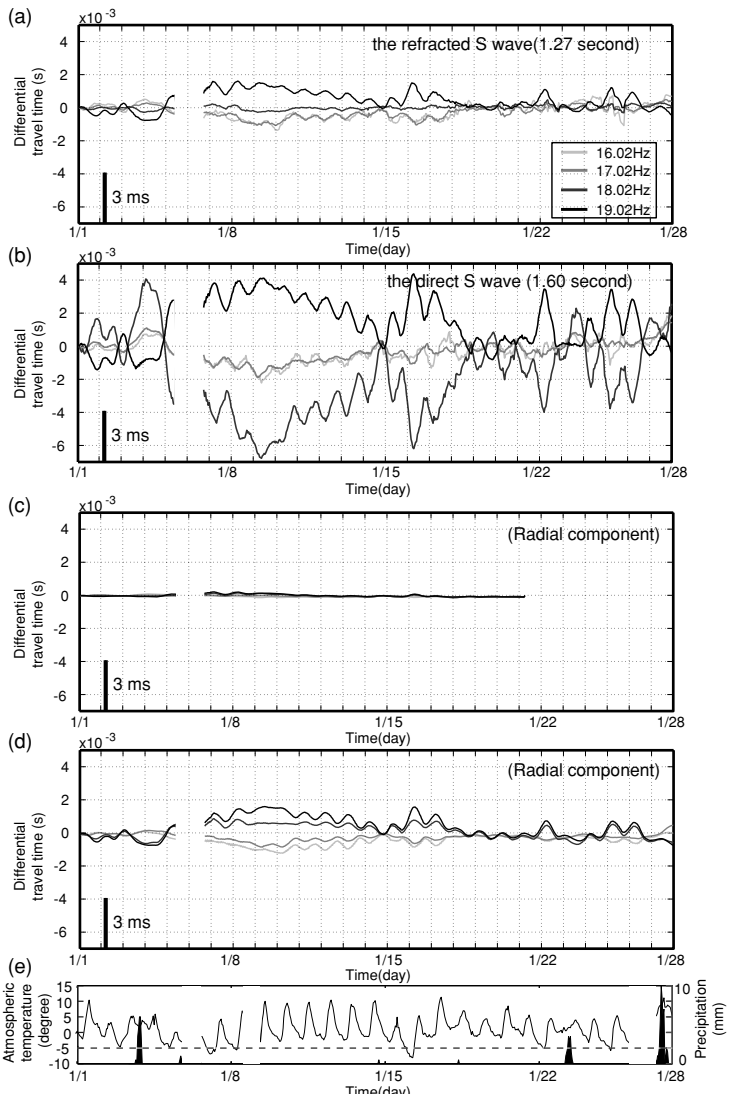

Fig. 12. Frequency dependence of temporal variations in differential travel time for the phases of (a) the refracted $S$-wave, (b) the direct $S$-wave, (c) radial motion of the source foundation and (d) the main wave with largest amplitude observed at the borehole seismometer (around $0.1 \mathrm{~s}$ ). The width of the frequency range from which we selected the signal components is $2 \mathrm{~Hz}$, which is centered at the frequency shown in the legend. This figure shows the temporal variations in the lower frequency band of 15.02-20.02 Hz. (e) Temporal variation in atmospheric temperature and precipitation.

between the source and receivers; it is likely that variation in the physical properties of the near-surface medium causes the observed variation in travel time.

\subsection{Direct $P$ - and $S$-waves}

Figure $8(\mathrm{~b})$ shows the temporal variation in differential travel time for the direct $P$ - and $S$-waves. These variations are statistically significant; the uncertainty of the travel time is within $0.1 \mathrm{~ms}$ for the observation period.

Variations in the direct $P$ - and $S$-waves are different from those of the refracted waves, with the variation in direct waves being less affected by the variation in atmospheric temperature than refracted waves. Marked changes in the differential travel time of direct waves are observed at times of rainfall. The observed changes involve an instantaneous delay in travel time followed by a gradual recovery. The amplitude of the change is $>1.0 \mathrm{~ms}$, and the change lasts 2 days or more. Such phase variations are not observed in the motion of the source foundation, nor in the temporal variation in apparent velocity at the array site. The effects of atmospheric temperature and barometric pressure are barely apparent in these data.
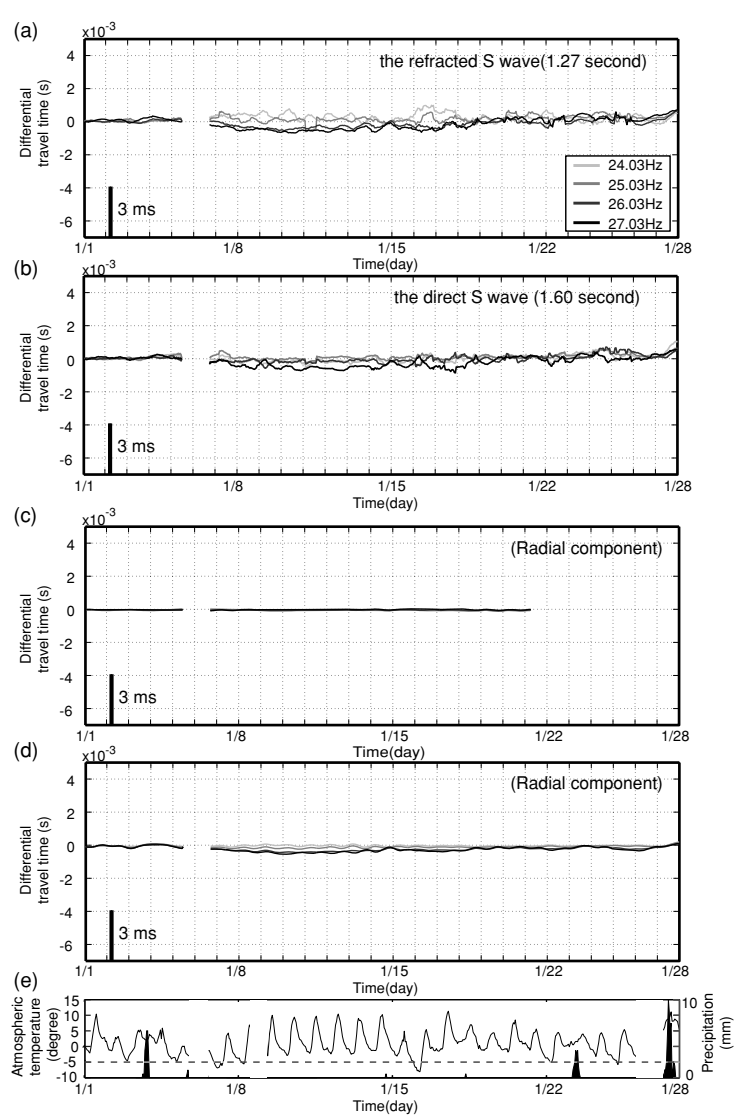

Fig. 13. Frequency dependence of temporal variations in differential travel time for the phases shown in Fig. 12(a)-(d). This figure shows the temporal variations in the frequency band of $23.03-28.03 \mathrm{~Hz}$. (e) Temporal variation in atmospheric temperature and precipitation.

\subsection{Surface wave and reflected waves}

Figure 11 shows the temporal variation in the surface wave and the phase that arrived at the array from a downward travel path. As the amplitudes of these phases were small, the data were stacked for $48 \mathrm{~h}$. Marked variation can be correlated with times of rainfall for the surface wave. The origin of this variation must also be related to rainfall along the path of the surface wave.

One of the advantages of array observation is in detecting the reflection of phases from deeper parts of the crust. From the data in Fig. 6, we can detect phases with high apparent velocity that are interpreted as representing the reflected wave that propagated from below. The data were also stacked over the elements of the array by assuming that the wave propagated vertically. As uncertainty is within 0.5 $\mathrm{ms}$ for the observation period, no marked variation can be correlated with other observation data.

\subsection{Frequency dependence of the temporal variation in differential travel time}

We investigated the frequency dependence of the temporal variation in differential travel time to take advantage of the frequency-discreteness of the ACROSS signal. Figure 12(a) and (b), and Figure 13(a) and (b) show the frequency dependence of the temporal variation in differential travel time for the refracted and the direct $S$-wave. In this analysis, the signal components in the specified range of frequency are selected from the stacked data set, and the 


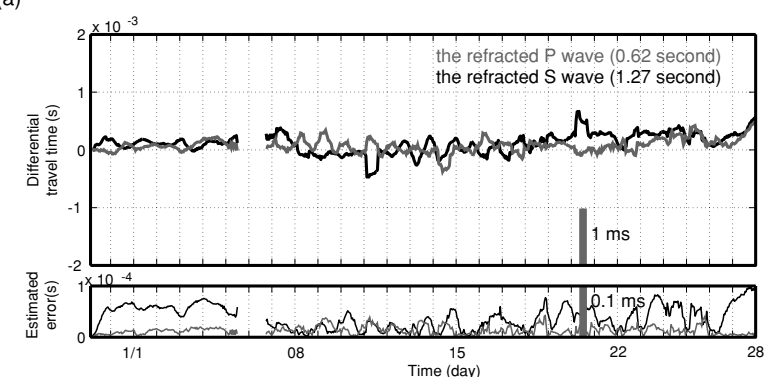

(b)
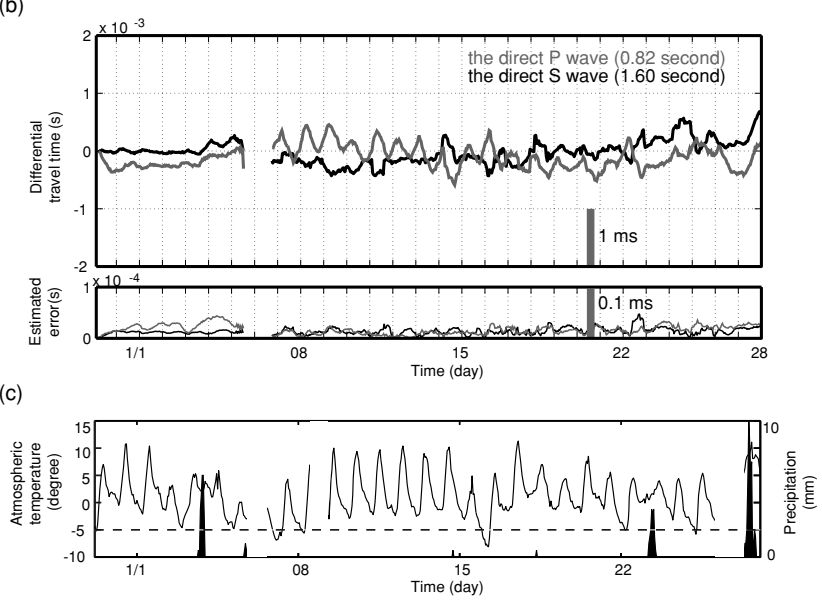

Fig. 14. Temporal variation of (a) the refracted $P$-wave and $S$ - waves, (b) the direct $P$-wave and $S$-waves except for a frequency band of 15-20 Hz. (e) Temporal variation in atmospheric temperature and precipitation.

differential travel times are estimated in the same way as Section 4.3. The width of the frequency range from which we selected the signal components is $2 \mathrm{~Hz}$, which is centered at the frequency shown in the legend. A strong frequency dependency is clearly observed in this figure. The temporal variation in the differential travel time appears to be sensitive to the variation in atmospheric temperature and rainfall, especially in the frequency range centered at 18.02 $\mathrm{Hz}$ and $19.02 \mathrm{~Hz}$. Although the variations show a similar pattern in the direct and the refracted $S$-waves, the amplitude of the variation is much larger in the direct $S$-wave. The differential travel times for the direct $S$-wave change in the opposite manner for $18.02 \mathrm{~Hz}$ and $19.02 \mathrm{~Hz}$ with respect to atmospheric temperature; therefore, the variations in travel time are cancelled out when averaged over all frequency components, and only a minor relationship appears for atmospheric temperature [Fig. 8(b)]. On the other hand, they change in the same manner with respect to rainfall, which is clearly seen for the rain on January 4, making the effect of rain remain after the variations in travel time have been averaged over all frequency components.

\section{Discussion and Conclusions}

In the investigation reported here we have attempted to identify a region that is affected by atmospheric temperature and rainfall using the vibration signal on the source foundation and at a borehole seismometer, which is about $50 \mathrm{~m}$ away from the ACROSS source. Figures 12(c) and (d) and 13(c) and (d) show the frequency dependence of a temporal variation in the radial motion of the source foundation and the initial part of the wave at the borehole seismometer. The variation at the borehole seismometer shows a similar pattern to the variation in the refracted $S$-wave and the direct $S$-wave. The amplitude of the variation at the borehole seismometer is comparable to that of the refracted $S$-wave. On the other hand, the amplitude at the source foundation is much smaller than that of the borehole seismometer. Thus, we may conclude that the main cause of the temporal variation exists in the region that is very close to the source. The reason why the variation in the direct $S$-wave is larger than the reflected $S$-wave may be attributed to the ray path of the direct $S$-wave, which travels through a relatively shallow part of the ground that can be more affected by the weather condition.

The variation in weather conditions, such as temperature and rainfall, contributes to the variation in the property of the medium near the surface. As the ACROSS vibrator is deployed on the surface, it may be strongly affected by the change in weather conditions. Saeki (2000) documented that compliance of the medium immediately surrounding the seismic source affects the energy of the radiated wave and its phase delay. This compliance is dependent upon the shear wave velocity in the medium around the source and the angular frequency of the force generated by the source. Once the angular frequency of the generated force is accurately controlled, we need to monitor shear wave velocity near the source, as this appears to vary with weather conditions.

The environmental effects can be removed from the waves to take advantage of the frequency-discreteness of the ACROSS signal. As the environmental effect is relatively large in the frequency between 15 and $20 \mathrm{~Hz}$, we removed the data corresponding to $15-20 \mathrm{~Hz}$ from our analysis. Figure 14 shows the temporal variation of the refracted wave and the direct wave, with the exception of a frequency band of $15-20 \mathrm{~Hz}$. We were able to remove the effect and obtain the travel time with less effect by environmental conditions.

We can investigate depth variation in elastic wave properties using various phases with different wave paths. In this study, we investigated depth variation using only direct and refracted waves, as there is a clear boundary in terms of elastic wave velocity at this site. If more boundaries exist, we can investigate more clearly the depth variations in the elastic wave properties of the rock medium, which might be expected to include cracks and possibly aquifers or similar features. Furumoto et al. (2001) compiled the stress sensitivity of a seismic wave velocity as a function of baseline length for the temporal variations related to tidal stress, tectonic stress and coseismic stress. The stress sensitivity over a baseline length is related to depth variations in the stress sensitivity because a seismic wave travels through deeper levels with an increase in epicentral distance. Velocity change is dependent on the degree of defect development in the rock mass, including cracks and pores, and this varies with depth. Furumoto et al. (2001) suggest that accurate and continuous monitoring over a long distance is required to determine the stress sensitivity of seismic wave velocity. An experiment using the ACROSS source is one of the most 
valuable methods of investigating the stress sensitivity.

Acknowledgments. We would like to acknowledge the use of data for elastic wave velocity by geophysical logging exploration carried out by the Tono Research Institute of Earthquake Science. We would also like to express our thanks to N. Fujii, T. Yamauchi and R. Ikuta for their useful comments. Reviews by Y. G. Li and P. G. Silver greatly improved the manuscript.

\section{References}

Alekseev, A. S., Chichinin, I. S. and Korneev, V. A., Powerful lowfrequency vibrators for active seismology, Bull. Seismol. Soc. Am., 95, 10-17, 2005.

Birch, F., The velocity of compressional waves in rocks to 10 kilobars, Part 1, J. Geophys. Res., 65, 1083-1102, 1960.

Bokelmann, G. H. R. and H.-P. Harjes, Evidence for temporal variation of seismic velocities within the upper continental crust, J. Geophys. Res., 105, 23,789-23,894, 2000.

Crampin, S., Geological and industrial implications of extensive dilatancy anisotropy, Nature, 328, 491-496, 1987.

Crampin, S., D. C. Booth, R. Evans, S. Peacock, and J. B. Fletcher, Changes in shear-wave splitting at Anza near the time of the North Palm Springs Earthquake, J. Geophys. Res., 95, 11,197-11,212, 1990.

Furumoto, M., Y. Ichimori, N. Hayashi, Y. Hiramatsu and T. Satoh, Seismic wave velocity changes and stress build-up in the crust of the KantoTokai region, Geophys. Res. Lett., 28, 3737-3740, 2001.

Hiramatsu, Y., N. Hayashi, M. Furumoto, and H. Katao, Temporal changes in coda Q-1 and b-value due to the static stress change associated with the 1995 Hyogo-ken Nanbu earthquake, J. Geophys. Res., 105, 6141$6151,2000$.

Ikuta, R. and K. Yamaoka, Temporal variation of shear wave anisotropy detected with ACROSS, J. Geophys. Res., 109, B09305, doi:10.1029/ 2003JB002901, 2004.

Ikuta, R., K. Yamaoka, K. Miyakawa, T. Kunitomo, and M. Kumazawa, Continuous monitoring of propagation velocity of seismic wave using ACROSS, Geophys. Res. Lett., 29, 13, 10.1029/2001GL013974, 2002.

Kumazawa, M. and Y. Takei, Active method of monitoring underground structures by means of ACROSS. 1. Purpose and principle, Abstr. Seismol. Soc. Jpn., 2, 158, 1994.

Kunitomo, T. and M. Kumazawa, Active monitoring of the Earth's structure by the seismic ACROSS-Transmitting and receiving technologies of the seismic ACROSS, Proc. 1st Int. Workshop Active Monitoring Solid Earth Geophysics, 181-184, 2004.

Li, Y.-G., J. E. Vidale, K. Aki, F. Xu, and T. Burdette, Evidence of shallow fault zone strengthening after the 1992 M7.7 Landers, California, earthquake, Science, 279, 217-219, 1998.
McEvilly, T. V. and L. R. Johnson, Stability of P and S velocities from central California quarry blasts, Bull. Seismol. Soc. Am., 64, 343-353, 1974.

Neidell, N. S. and M. T. Taner, Semblance and other coherency measures for multichannel data, Geophysics, 36, 486-497, 1971.

Niu, F., P. G. Silver, R. M. Nadeau, and T. V. McEvilly, Migration of seismic scatterers associated with the 1993 Parkfield aseismic transient event, Nature, 426, 544-548, 2003.

Nur, A., Dilatancy, pore fluids and premonitory variations of ts/tp travel times, Bull. Seismol. Soc. Am., 62, 1217-1222, 1972.

Reasenberg, P. and K. Aki, A precise, continuous measurement of seismic velocity for monitoring in situ stress, J. Geophys. Res., 79, 5412-5426, 1974.

Saeki, M., Theoretical study of the ability of the ACROSS source to generate seismic wave field, MSc., University of Tokyo, Tokyo 2000.

Saiga, A., Y. Hiramatsu, T. Ooida, and K. Yamaoka, Spatial variation in the crustal anisotropy and its temporal variation associated with the moderate size earthquake in the Tokai region, central Japan, Geophys. J. Int., 154, 695-705, 2003.

Sato, H., Temporal change in scattering and attenuation associated with the earthquake occurrence-A review of recent studies on coda waves, Pure Appl. Geophys., 126, 465-497, 1988.

Scholz, C. H., M. Wyss, and Y. P. Aggarwal, The physical basis for earthquake prediction, Science, 181, 803-810, 1973.

Semenov, A. N., Variation in the travel-time of transverse and longitudinal waves before violent earthquakes, Bull. Acad. Sci. USSR, Phys. Solid Earth, 3, 245-248, 1969.

TRIES, Geophysical log of TGR350; Tono Research Institute of Earthquake Science, 2001.

Yamamura, K., O. Sano, H. Utada, Y. Takei, S. Nakao, and Y. Fukao, Longterm observation of in situ seismic velocity and attenuation, J. Geophys. Res., 108, B6, 2317, 10.1029/2002JB002005, 2003.

Yamaoka, K. and R. Ikuta, Feasibility of reflection monitoring for plate coupling using ACROSS System, Proceedings of the 1st International Workshop on Active Monitoring in the Solid Earth Geophysics, 126129, 2004.

Yamaoka, K., T. Kunitomo, K. Miyakawa, K. Kobayashi, and M. Kumazawa, A trial for monitoring temporal variation of seismic velocity with ACROSS system, Island Arc, 10, 336-347, 2001.

Yoshida, Y., H. Ueno, and Y. Ishikawa, The observation and analysis of ACROSS signals by seismic networks-Determination of the transfer function. Proc. 1st Int. Workshop Active Monitoring Solid Earth Geophysics, 258-261, 2004.

A. Saiga (e-mail: saiga@sevo.kyushu-u.ac.jp), K. Yamaoka, T. Kunitomo, and T. Watanabe 